Originally published as:

Mousset, S., Bug, G., Heinz, W.J., Tintelnot, K., Rickerts, V.

Breakthrough zygomycosis on posaconazole prophylaxis after allogeneic stem cell transplantation

(2010) Transplant Infectious Disease, 12 (3), pp. 261-264.

DOI: 10.1111/j.1399-3062.2009.00479.x

The definitive version is available at: http://onlinelibrary.wiley.com/ 


\title{
Breakthrough zygomycosis on posaconazole prophylaxis after allogeneic stemcell transplantation
}

\author{
S. Mousset ${ }^{1}$, G. Bug ${ }^{1}$, W.J. Heinz ${ }^{2}$, K. Tintelnot ${ }^{3}$, V. Rickerts ${ }^{1}$ \\ ${ }^{1}$ Medizinische Klinik II, Klinikum der J.W. Goethe-Universität, Frankfurt am Main, Germany \\ ${ }^{2}$ Medizinische Klinik und Poliklinik II, Universitätsklinikum Wuerzburg, Wuerzburg, Germany \\ ${ }^{3}$ Robert Koch-Institut, Berlin, Germany
}

\begin{abstract}
Antifungal prophylaxis with posaconazole (POS) has been shown to decrease the mortality associated with invasive fungal infections in high-risk patients. We report on a patient, with severe graft-versushost disease after allogeneic stem cell transplantation, who developed proven pneumonia due to Rhizopus microsporus after 40 days of POS prophylaxis (fasting serum levels: $691^{\wedge} 904 \mathrm{ng} / \mathrm{mL}$ ).

Despite combination treatment with liposomal amphotericin B and POS for 39 days, the patient died from pulmonary hemorrhage. This case highlights the need for continued awareness of breakthrough zygomycosis in patients receiving POS.
\end{abstract}

Invasive fungal infections remain a major complication in patients with graft-versus-host disease (GVHD) after allogeneic stem cell transplantation. The administration of posaconazole (POS) has been shown to reduce the mortality attributable to invasive fungal infections in these highrisk

patients (1).Very few breakthrough infections, mainly due to Aspergillus and Candida species, have been described in the initial trial (1). So far only 1 case of breakthrough infection due to Zygomycetes species on POS prophylaxis has been documented in the literature (2).

\section{Case report}

In August 2007, a 60-year-old male patient received an allogeneic stem cell graft for acute myelogenous leukemia. He had no history of a prior invasive fungal infection and there were no major infectious complications before engraftment. The patient was readmitted on day 95 after transplantation because of intestinal GVHD (grade III). He was treated with prednisolone, mycophenolate mofetil, pentostatin, and cyclosporin A (which was later switched to tacrolimus). This treatment resulted in improvement of the symptoms of GVHD but was complicated by steroidinduced diabetes mellitus (without lactic acidosis) and worsening of a preexisting chronic renal insu/ciency. At the time of diagnosis of GVHD, the patient complained about a dry cough, and a computed tomography (CT) scan of the chest was performed. It showed a lesion with a peripheral halo in the anterior segment of the right upper lobe suggestive of an invasive mold infection. Because of the reduced general condition of the patient, he was treated with voriconazole (intravenous, then oral) without further invasive diagnostics for microbiological confirmation of the suspected invasive aspergillosis. The dry cough completely resolved and a follow-up CT scan on day 1164 showed residual scarring of the lesion.Voriconazole treatment was stopped on day 1188 after transplantation and POS (200mg 3 times a day) was started as secondary prophylaxis. The patient was discharged with stable symptoms of GVHD on continued immunosuppressive treatment. Despite persistent diarrhea, tacrolimus blood levels indicated adequate resorption of oral drugs. POS trough levels, determined by a high-performance liquid chromatographic assay performed on stored frozen fasting blood samples obtained on days 25 and 35 of POS prophylaxis, were 691 and $904 \mathrm{ng} / \mathrm{mL}$.

On day 40 of POS prophylaxis, the patient was readmitted with worsening fatigue, a dry cough, and elevated Creactive protein $(41 \mathrm{mg} / \mathrm{L}$; normalo5mg/L). The blood cell count showed pancytopenia (leukocytes $1.4 \times 10^{9} / \mathrm{L}$, haemoglobin $5.9 \mathrm{~g} / \mathrm{dL}$, and platelets $20 \times 10^{9} / \mathrm{L}$ ) probably due to valganciclovir treatment for cytomegalovirus reactivation. A relapse of leukemiawas thought to be unlikely because of donor chimerism of $100 \%$ in the peripheral blood. A CT scan of the chest showed a new mass lesion in the posterior segment of the right upper lobe (Fig. 1a). The scar in the anterior segment of the 
right upper lobe was unchanged. POS was stopped, and caspofungin and broad-spectrum antibacterials were initiated.

On day 5 after admission, bronchoscopy revealed a necrotic mucosal lesion of the corresponding bronchus. Histology from that lesion revealed broad, unseptated hyphae suggestive for zygomycosis. Antifungal therapy was changed to liposomal amphotericin B (AmB) $(3 \mathrm{mg} / \mathrm{kg})$ on day 6 . A zygomycete was cultured from bronchoalveolar lavage, phenotypically identified as Rhizopus microsporus. This was confirmed by sequencing of the ITS regions of ribosomal DNA (3). In vitro susceptibility testing, using the CLSI M38-A method, revealed minimal inhibitory concentrations (MIC) of $8 \mathrm{mg} / \mathrm{mL}$ for voriconazole, $2 \mathrm{mg} / \mathrm{mL}$ for POS, $416 \mathrm{mg} / \mathrm{mL}$ for caspofungin, and $1 \mathrm{mg} /$

$\mathrm{mL}$ forAmB.

Markers of inflammation rapidly declined after initiation of liposomal AmB and the patient's clinical condition gradually improved. A follow-up CTscan of the chest on day 23 showed a cavitation of the lesionwith progressive signs of perifocal bleeding (Fig. 1b). Resection of the lesion was precluded by severe transfusion-refractory thrombocytopenia. POS (400 mg twice daily) was added to liposomal AmB on day 27 after admission. The serum trough level of POS one week later was $907 \mathrm{ng} / \mathrm{mL}$. Despite a stable lesion in a second follow-up CT scan on day 48 after admission, the patient died on day 68 from a massive pulmonary hemorrhage secondary to pulmonary zygomycosis and severe thrombocytopenia

\section{Discussion}

This case of breakthrough zygomycosis during POS prophylaxis highlights the need for continued awareness of invasive zygomycosis in patients receiving mold active antifungal prophylaxis. It documents the importance of a rapid diagnostic workup to facilitate active antifungal therapy and shows the limitations of current antifungal treatment regimens in persistently immunocompromised patients.

Tissue levels of POSwere apparently not sucient to prevent zygomycosis in this patient. Although target plasma levels have not yet been defined, levels determined in this patient suggest absorption despite intestinal GVHD. A dose-dependent antifungal e/ciency is suggested by 2 findings. First, higher POS serum levels were associated with higher response rates in the setting of salvage therapy for invasive aspergillosis (4). Second, pharmacokinetic data from the prophylaxis in GVHD trial showed a trend to lower plasma levels in 5 patients with breakthrough fungal infections compared with patients without fungal infections (median Caverage POS level of $611 \mathrm{vs.} 922 \mathrm{ng} / \mathrm{mL}$ ) (5). We determined trough levels, which are similar to average levels, as POS achieves a steady state after $7^{\wedge} 10$ days (5).

Several reports suggest an association of long-term voriconazole administrationwith invasive zygomycosis, especially in recipients of allogeneic stem cell transplants (6^8). Although selective pressure appears to be aplausible explanation for this association, recent data from a mice model of Rhizopus oryzae infection suggest that pre-exposure to voriconazole may enhance the virulence of zygomycetes (9).

Rapid initiation of in vitro active antifungals is of paramount importance in patients with zygomycosis. In an analysis of 70 patients with hematologic malignancies and zygomycosis, start of AmB treatment beyond day 6 after the onset of symptoms was associated with a poor outcome

(10). In the case presented here, caspofungin was given because of pre-existing renal insurciency and suspected invasive aspergillosis due to malabsorption of POS. Antifungal therapy with caspofungin alone is inactive in zygomycosis and should not be used in patients with suspected mold infections during POS prophylaxis unless zygomycosis is ruled out.

The in vitro activity of POS varies for different zygomycetes. Because interpretive breakpoints for susceptibility testing of POS are missing, approved breakpoints for fluconazole and voriconazole testing against Candida species have been taken as surrogate markers (11).Therefore, POS MIC have to be interpreted with caution. A mouse model suggests that, in contrast to other zygomycetes, higher doses of POS might be needed to treat infections due to R. microsporus (12).

Optimal outcome of zygomycosis requires the reversal of underlying conditions, early antifungal therapy with highdose liposomal $\mathrm{AmB}$, and surgical resection of involved tissue. In our patient, the 
underlying condition could not be reversed, surgery was not possible owing to transfusion-resistant thrombocytopenia, and preexisting renal insufficiency precluded high-dose AmB. Therefore, we used antifungal combination therapy when pulmonary lesions did not improve on AmB therapy on follow-up CT scans. The combination of AmB and POS is supported by a synergistic effect on hyphae of zygomycetes in vitro (13), in an animal model (14), and previous anecdotal experience in our center (15).

Taken together, the reasons for treatment failure in this patientmight be insucient tissue levels of POS in necrotic lung tissue that could not be resected by surgery in combination with a state of severe and irreversible immunosuppression. Newer antifungal treatment approaches, such as chelation therapy that is currently under clinical investigation (16), are needed for the treatment of zygomycosis in persistently immunocompromised patients.

\section{References}

1. Ullmann AJ, Lipton $\mathrm{JH}$, Vesole $\mathrm{DH}$, et al. Posaconazole or fluconazole for prophylaxis in severe graft-versus-host disease. N Engl J Med 2007; 356: 335^347.

2. Schlemmer F, Lagrange-XelotM, Lacroix C, de LaTR, Socie G,Molina JM. Breakthrough Rhizopus infection on posaconazole prophylaxis following allogeneic stem cell transplantation. Bone Marrow Transplant 2008; 42: 551^552.

3. Schwarz P, Bretagne S, Gantier JC, et al. Molecular identification of zygomycetes from culture and experimentally infected tissues. J Clin Microbiol 2006; 44: 340^349.

4. WalshTJ, Raad I, PattersonTF, et al.Treatment of invasive aspergillosis with posaconazole in patients who are refractory to or intolerant of conventional therapy: an externally controlled trial. Clin Infect Dis 2007; 44: 2^12.

5. Krishna G, Martinho M, Chandrasekar P, Ullmann AJ, Patino $H$. Pharmacokinetics of oral posaconazole in allogeneic hematopoietic stem cell transplant recipients with graft-versus-host disease. Pharmacotherapy 2007; 27: 1627^1636.

6. Marty FM, Cosimi LA, Baden LR. Breakthrough zygomycosis after voriconazole treatment in recipients of hematopoietic stem-cell transplants. N Engl J Med 2004; 350: 950^952.

7. Trifilio S, Singhal S, Williams S, et al. Breakthrough fungal infections after allogeneic hematopoietic stemcell transplantation in patients on prophylactic voriconazole. Bone MarrowTransplant 2007; 40: $451^{\wedge} 456$

8. Trifilio SM, Bennett CL,Yarnold PR, et al. Breakthrough zygomycosis after voriconazole administration among patients with hematologic malignancies who receive hematopoietic stem-cell transplants or intensive chemotherapy. Bone MarrowTransplant 2007; 39: 425^429.

9. Lamaris GA, Ben-Ami R, Lewis RE, Chamilos G, Samonis G, Kontoyiannis DP. Increased virulence of Zygomycetes organisms following exposure to voriconazole: a study involving fly and murine models of zygomycosis. J Infect Dis 2009; 199: 1399^1406.

10. Chamilos G, Lewis RE, Kontoyiannis DP. Delaying amphotericin Bbased frontline therapy significantly increases mortality among patients with hematologic malignancy who have zygomycosis. Clin Infect Dis 2008; 47: 503^509.

11. Pfaller MA, Messer SA, Boyken L,Tendolkar S, Hollis RJ, Diekema DJ. Selection of a surrogate agent (fluconazole or voriconazole) for initial susceptibility testing of posaconazole against Candida spp.: results from a global antifungal surveillance program. J Clin Microbiol 2008; 46: 551^559.

12. Dannaoui E, Meis JF, Loebenberg D,Verweij PE. Activity of posaconazole in treatment of experimental disseminated zygomycosis. Antimicrob Agents Chemother 2003; 47: 3647^3650.

13. Perkhofer $S$, Locher $M$, Cuenca-Estrella $M$, et al. Posaconazole enhances the activity of amphotericin B against hyphae of zygomycetes in vitro. Antimicrob Agents Chemother 2008; 52: $2636^{\wedge} 2638$.

14. RodriguezMM, Serena C,MarineM, Pastor FJ, Guarro J. Posaconazole combined with amphotericin B, an effective therapy for a murine disseminated infection caused by Rhizopus oryzae. Antimicrob Agents Chemother 2008; 52: 3786^3788.

15. RickertsV, Atta J, Herrmann S, et al. Successful treatment of disseminated mucormycosis with a combination of liposomal amphotericin B and posaconazole in a patient with acute myeloid leukaemia. Mycoses 2006; 49 (Suppl 10): 27^30.

16. Reed C, Ibrahim A, Edwards JE Jr,Walot I, Spellberg B. Deferasirox, an iron-chelating agent, as salvage therapy for rhinocerebral mucormycosis. Antimicrob Agents Chemother 2006; 50: 3968^3969. 
Figure 1 (a) Computed tomography (CT) of the chest on admission showing a mass lesion in the right upper lobe. (b) Follow-up CT on day 23 showing cavitation of the lesion and signs suggestive of perifocal bleeding.
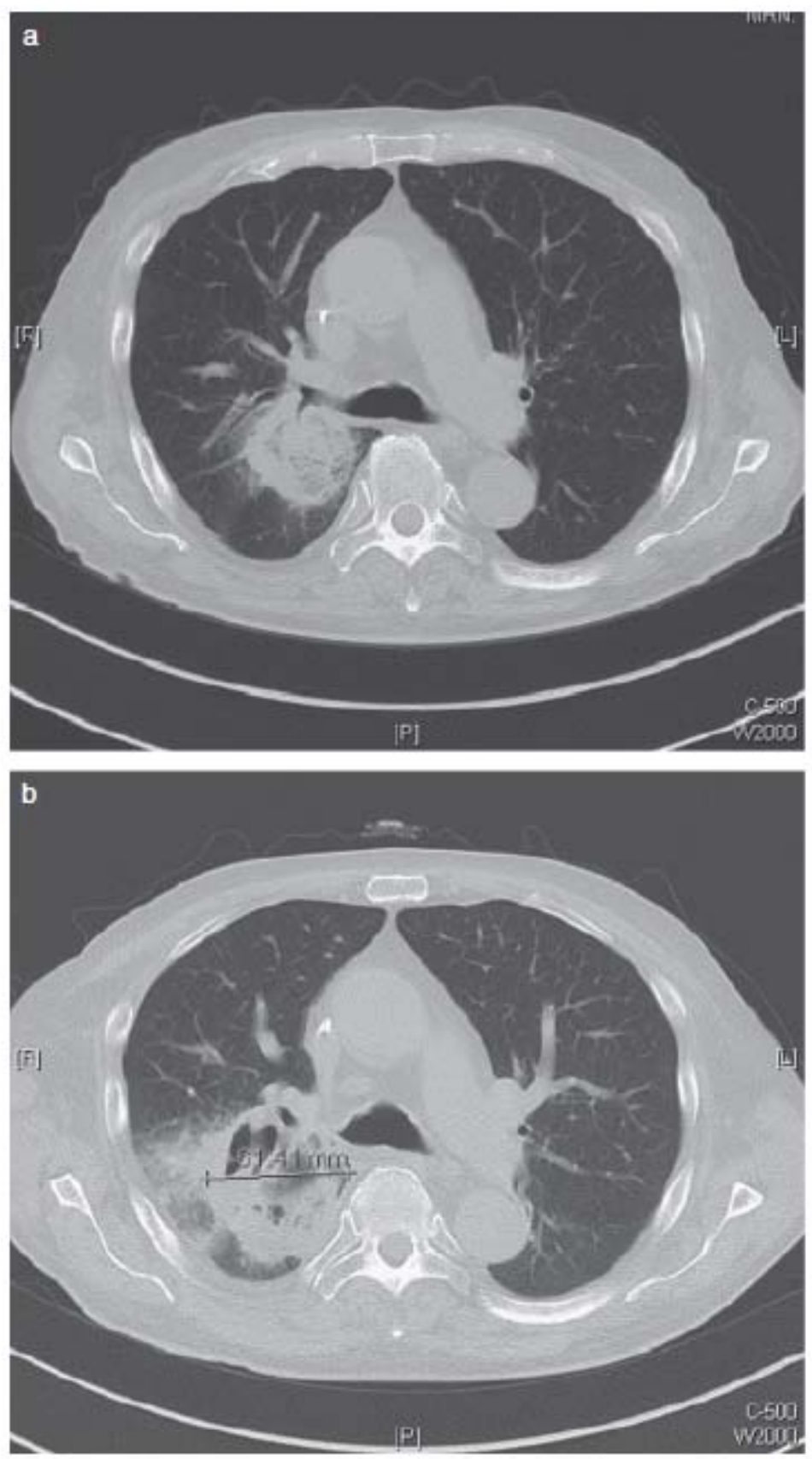